\section{Respuesta de los autores a la réplica al artículo «influencia estacional en las características de los pacientes ingresados en una unidad de cuidados intensivos»}

\section{Response of the authors to the reply to the article: "Seasonal influence in characteristics of patients admitted to an intensive care unit"}

\section{Sr. Director:}

Nos resulta gratificante el que otros autores hayan aplicado la misma hipótesis de trabajo en su medio, basándose en nuestro artículo publicado en Medicina Intensiva ${ }^{1}$.

En respuesta a los autores del trabajo realizado por Molina-González E et al, en primer lugar, decirles que al no disponer de los datos de la población estudiada por ellos, no podemos compararlo con el estudio efectuado por nuestro grupo. Los resultados del estudio llevado a cabo en nuestro hospital, donde se atiende a toda la población crítica que ingresa, puede no ser extrapolable a otros hospitales donde no se atiendan ciertas patologías críticas.

De todas formas, nos resultan muy interesantes los resultados de su estudio, lo que nos hace pensar que a pesar de la diferencia de localización entre las dos poblaciones estudiadas ( $14^{\circ}$ de latitud y $17^{\circ}$ de longitud), el efecto de la influencia climática en la enfermedad crítica quizá puede estar amortiguada por las medidas de aclimatación modernas con las que cuenta la población en su hábitat, hoy día. Todo esto abre una serie de interrogantes, que precisan de estudios epidemiológicos más amplios en los que habrá que considerar los recursos de la sociedad moderna.

Con respecto al método estadístico utilizado decirles que, en efecto, hemos utilizado la regresión logística binaria puesto que comparamos las estaciones de mayor oscilación térmica.

\section{Bibliografía}

1. Santana Cabrera L, Sánchez-Palacios $M$, Uriarte Rodriguez $A$, Fernández Arroyo M, Martínez Cuéllar S, Lorenzo Torrent R. Influencia estacional en las características de los pacientes ingresados en una unidad de cuidados intensivos. Med Intensiva. 2010;34:102-6.

\section{Santana Cabrera* y M. Sánchez-Palacios}

Servicio de Medicina Intensiva del Hospital Universitario Insular de Gran Canaria, Gran Canaria, España

*Autor para correspondencia.

Correo electrónico: Isancabx@gobiernodecanarias.org

(L. Santana Cabrera).

Véase contenido relacionado en DOI: 10.1016/j.medin.2010. 04.005

\section{A 40 años de la descripción del catéter de Swan Ganz}

\section{0 years after the description of the Swan Ganz Catheter}

Lopez A et $\mathrm{al}^{1}$ hacen una excelente revisión de las indicaciones y necesidades del catéter de flotación pulmonar en las unidades de cuidados intensivos, aunque aun existen voces que cuestionan su utilidad, las personas que hemos recibido entrenamiento en el manejo del mismo, alabamos no cuestionamos su uso, es importante que la nuevas generaciones de médicos en formación en terapia intensiva se les instruya a la colocación, manejo e interpretación del mismo, para el bien de nuestros pacientes y acuciosidad del clínico.

\section{Bibliografía}

1. Carrillo López A, Fiol Sala M, Rodríguez Salgado A. El papel del catéter de Swan-Ganz en la actualidad. Med Intensiva. 2010;34:203-14.

\section{J.L. Sandoval Gutiérrez}

Departamento de Medicina Crítica, Instituto Nacional de Enfermedades Respiratorias, México D.F., México Correo electrónico: sandovalgutierrez@gmail.com 